\title{
Comparison of Different Molecular Subtypes with $14 \%$ Ki-67 Cut-off Threshold in Breast Cancer Patients of Pakistan- An Indication of Poor Prognosis
}

\author{
Mehreen Mushtaq, FCPS ${ }^{1,2 *}$; Summaya Sohail Chaudry, MD ${ }^{1 \# ;}$ Ahmareen Khalid Sheikh, FCPS ${ }^{1}$; Nazia Khan, FCPS ${ }^{1}$; Asma Khattak, \\ MPhil'; Aisha Akbar, MD³; Ashok Kumar Tanwani, MPhil'; Tanwir Khaliq, FCPS4; Muhammad Faraz Arshad Malik, PhD5; Syeda \\ Kiran Riaz, PhD ${ }^{1,5,6 *}$ \\ ${ }^{1}$ Department of Pathology, Shaheed Zulfiqar Ali Bhutto Medical University, Pakistan Institute of Medical Sciences, Islamabad, Pakistan \\ ${ }^{2}$ Armed Forces Institute of Pathology, Rawalpindi, Pakistan \\ ${ }^{3}$ Rawal Institute of Health Sciences, Islamabad, Pakistan \\ ${ }^{4}$ Department of Surgery, Shaheed Zulfiqar Ali Bhutto Medical University, Islamabad, Pakistan \\ ${ }^{5}$ Department of Biosciences, COMSATS University Islamabad, Pakistan \\ ${ }^{6}$ Department of Molecular Biology, Shaheed Zulfiqar Ali Bhutto Medical University, Islamabad, Pakistan \\ *Shared first authorship
}

\begin{abstract}
Background: $\mathrm{Ki}-67$ is a proliferation marker that is used not only to categorize patients in luminal A and B subtypes of breast cancers, but also to determine the aggressiveness of the disease in triple negative and human epidermal growth factor 2 (HER2) over expressed molecular subtypes. The present study was designed to evaluate the role of Ki-67 with cut off value of $14 \%$ in molecular subgroups and its association with patient prognosis.

Methods: Immunostaining was performed on histopathologically confirmed sections $(n=278)$ to assess expression of Ki-67, estrogen receptor (ER), progesterone receptor (PR) and HER2. Immunoreactivity of molecules was recorded as percentage scoring. Results: Adopting a cut off value of $14 \%$, Ki- 67 was high in $88 \%$ of the cases included in the study. High Ki- 67 was significantly associated with pathological parameters including histological grade, advanced stage and nodal/distant metastasis. Immunoexpression of ER, PR and HER2 also showed strong correlation with high expression of Ki-67. Based on the St. Gallen classification, the cases were categorized into luminal A (10\%) and luminal B (51\%), triple negative (20\%) and HER2 enriched (18\%). Ki-67 index was also significantly high in 98\% of HER2 enriched and 95\% of TNBC patients. Interestingly, Ki-67 score with cut off value of $14 \%$ proved to be significant in deciphering prognosis in luminal patients. Moreover, high expression of Ki-67 also proved to be a marker of poor prognosis, especially in triple negative patients.

Conclusion: We suggest that utilization of IHC4 status i.e. ER, PR, HER2 and Ki-67 along with pathological findings and molecular subtyping can considerably affect clinical as well as therapeutic decisions.

Keywords: Breast cancer, IHC4, Ki-67 index, Molecular subtypes, Prognosis

Cite this article as: Mushtaq M, Chaudry SS, Khalid Sheikh A, Khan N, Khattak A, Akbar A, et al. Comparison of different molecular subtypes with $14 \%$ ki-67 cut-off threshold in breast cancer patients of pakistan- an indication of poor prognosis. Arch Iran Med. 2021;24(11):837-844. doi: 10.34172/aim.2021.124
\end{abstract}

Received: October8, 2020, Accepted: December15, 2020, ePublished: November 1, 2021

\section{Introduction}

Breast cancer is the most frequent cause of cancer-related death in females in underdeveloped countries because of lack of screening strategies and limited treatment facilities. ${ }^{1}$ The main prognostic markers for breast cancer are size of tumor, lymph node status, distant metastasis and histological grade. Over the past decade, there have been great advances in classification of breast cancer ranging from histopathological to molecular subtypes. ${ }^{2}$

Immunohistochemistry (IHC) is vastly used to aid in therapeutic decision making for precision medicine and improving patient prognosis. The IHC4 panel consisting of estrogen receptor (ER), progesterone receptor (PR), human epidermal growth factor 2 receptor (HER2) and Ki-67 (proliferation index marker) is used for patient stratification in different molecular subtypes. ${ }^{3}$ According to the St. Gallen consensus conference (2011), the expert panel simplified the therapy indications among breast cancer subtypes based on these 4 biomarkers. The intrinsic classification includes luminal type $\mathrm{A}$ and $\mathrm{B}$, Her-2 enriched and triple negative subtype. The majority of breast tumors are of luminal type expressing hormone receptors, whereas luminal B is categorized with high Ki-67 index. Therefore, luminal B demonstrates aggressiveness and has worse recurrence free survival while luminal A carries better prognosis. ${ }^{4}$ The HER-2 enriched subtype comprises $15-25 \%$ of tumors and predicts response to trastuzumab. Basal like/triple negative tumors make up $10-20 \%$ of all breast cancers with poor prognosis due to lack of targeted therapy. The incidence, response to treatment, prognosis and survival are different for each molecular subtype. ${ }^{5}$

$\mathrm{Ki}-67$ is a nuclear protein that reflects cell proliferation 
and is identified in various steps of cell cycle except for the resting stage. ${ }^{6}$ The most well-known method of Ki-67 analysis is immunostaining. Staining of Ki-67 is variable and pathologists count hundreds of cells to calculate the percentage by taking into account nuclear staining. ${ }^{7}$ It is an important marker for defining the molecular subtype of breast cancer. Molecular subtyping is essentially important in exploring novel therapies for metastatic, resistant and recurrent cases. ${ }^{8}$ Several studies showed that high levels of Ki-67 predicted better response to chemotherapy as adjuvant therapy. ${ }^{9}$ The guidelines for Ki-67 scoring are still under development. It is of importance as it helps to differentiate between Luminal A and Luminal B subtypes by adopting the cut off value of $14 \% .{ }^{10}$ The panel agreed on indication of endocrine therapy in clinic-pathologically classified Luminal A as it was found to be less responsive to chemotherapy. Chemo-endocrine therapy in Luminal B subtype has been suggested in HER2 positive cases while chemotherapy is recommended for patients with triple negative disease. ${ }^{11}$ The rationale of this study is to see the frequency of Ki-67 in breast cancer in our population with $14 \%$ cut-off threshold due to variability in Ki-67 index in previously published data. Moreover, it is important to differentiate between Luminal $\mathrm{A}$ and Luminal $\mathrm{B}$ with recommended Ki-67 index as they carry different prognoses and may better aid the clinician in deciding about the treatment option.

\section{Materials and Methods Collection of Samples}

This study was conducted in the Department of Pathology, Shaheed Zulfiqar Ali Bhutto Medical University and its affiliated hospital, Pakistan Institute of Medical Sciences (PIMS), Islamabad. Sample size was calculated using the World Health Organization (WHO) Sample Size Calculator based on the representative breast cancer affected population and patients visiting department of Pathology, PIMS hospital annually. Tumor tissues (n = 278) were collected in 2016 with a follow-up period of 38 months. Ethical approvals were obtained from the university as well as the hospital and consent letters were acquired from patients before sample collection. Information regarding demographic data was obtained by interviewing patients. All non-epithelial tumors, atypical ductal hyperplasia, DCIS and LCIS, lesion reported as suspicious for malignancy on Histopathology (B-3/B-4) as well as male patients were excluded from the study.

\section{Tissue Processing}

Immediately after surgery, tissues were immersed in $10 \%$ buffered formalin and processed in automated tissue processor (LEICA TP-1020, IL, USA). Tissue processing was followed by preparation of paraffin embedded blocks, slides and hematoxylin and eosin (H\&E) staining. Breast cancer tissues were graded according to the Nottingham scoring system that includes extent of tubular differentiation in carcinoma, mitotic figures and nuclear pleomorphism. CX22 LED, Olympus microscopes (Tokyo, Japan) were used in the interpretation. Mitosis cut off was calculated for the field diameter of the microscope. The reporting pathologist staged the tumor by TNM staging incorporating tumor size, lymph node involvement and distant metastasis. ${ }^{12}$

\section{Immunohistochemistry}

IHC was performed using the 1 step horse reddish peroxidase (HRP) technique. A single slide from each case was selected for staining and histological evaluation. Tissue sections were mounted on coated slides. The slides were deparaffinized and hydrated in water. The slides underwent antigen retrieval at $\mathrm{pH} 9$ using the pressure cooker technique. Primary antibody for ER (PA0151), PR (PA0321), HER2 (PA0571) and Ki-67 (PA0230) (Leica Bond, IL, USA) were applied followed by application of DAB (3,3'-Diaminobenzidine, Sigma-Aldrich, Darmstadt, Germany). The slides were than stained with hematoxylin and eosin. Sections from lymph node germinal center were used as a positive control for Ki-67. Normal breast tissue was considered as control. Nuclear staining was taken as positive for ER, PR and Ki-67. Hot spot areas were selected at low power where staining was maximum. Markers were expressed as percentage of positively stained cells among total number of tumor cells at high power magnification (40X). At least 100 malignant cells were counted in representative sections. ${ }^{13}$ The staining of $\mathrm{Ki}-67$ was considered low if the percentage was less than $14 \%$ and high, if the percentage was more than $14 \%$.

Immunohistochemical interpretation was done by two specialized histopathologists using the Allred scoring system. It is an internationally recognized scoring system for estrogen and PR interpretation and utilizes two tiers, proportion score (PS) and intensity score (IS). The total score is calculated by adding the two scores. Scores of 3 or more were considered as positive. Similarly, interpretation of HER 2 was done taking $10 \%$ of membranous staining as cut-off. ${ }^{12}$

\section{Data Analysis}

Data was analyzed using SPSS version 21 and GraphPad version 5. Qualitative variables such as histologic type, grade, ER, PR and Her-2 were expressed in terms of frequency and percentage. Mean and standard deviation were calculated for quantitative variables like Ki-67 index and age of patients. Post-stratification, chi-square test or Fisher's exact test were applied wherever feasible and a $P$ value of $\leq 0.05$ was considered significant. Kaplan-Meier analysis and log rank test $(n=278)$ were performed (follow-up started from February, 2016) and hazard ratios (95\% confidence interval) were calculated for survival analysis. The mean follow-up period for survival analysis was 24 months with a median value of 27 months and an interquartile range of 4-36 months.

Results

Demographic and Clinicopathological Data 
The age range was 20 to 87 years and the median age of patients in this study was $47 \pm 12$ years. Almost $51 \%$ of the patients were below the median age with $26 \%$ in their $2^{\text {nd }}$ and $3^{\text {rd }}$ decade, showing greater prevalence of early disease onset. The majority of patients (62\%) included in the study exhibited pre-menopausal status. Invasive ductal carcinoma was the most common histological type (98\%). Three cases of invasive lobular carcinoma (1\%) were included, all of whom were grade I, 3 cases of invasive metaplastic carcinoma were also included with high grade.

\section{Biomarker Status and Molecular Subtyping}

Biomarkers included in the study were ER, PR, HER2 and Ki-67 with percent positivity of $61,46,50$ and $88 \%$ respectively while the mean $\mathrm{Ki}-67$ index was found to be $44 \%$. The range of $\mathrm{Ki}-67$ index was 1 to $95 \%$ with a standard deviation of 25.2. The majority of ER/PR positive patients presented with initial grades, lower invasive potential having the reverse trend for HER2 and Ki-67. Moreover, high Ki-67 index $(>14)$ was observed in ER (83\%), PR (82\%) and HER2 (96\%) positive cases (Figure 1). In comparison to ER/PR positive cases, HER-2 positive samples exhibited significantly greater odds of having higher Ki-67 index in breast cancer affected patients. Following the St. Gallen international conference, the cohort was divided in four intrinsic molecular subtypes based on the expression profile of ER, PR, HER2 and Ki67. Cases having ER and/or PR+ve, HER2-ve and Ki-67 low $(<14 \%)$ were considered as luminal A while patients having ER and/or PR+ve, HER2 any and Ki-67 high were categorized as luminal B. Patients were classified as HER2 enriched based on a molecular profile having ER and PRve, HER2+ve and Ki-67 any while triple negative patients lacked the expression of ER, PR and HER2. High Ki-67 index was observed in $98 \%$ of HER2 enriched and $96 \%$ of patients having the TNBC subtype (Figure 2).

\section{Correlation of Ki-67 with tumor Grade and Stage}

The majority of patients presented with higher grades $(85 \%)$ and advanced stages (39\%). Almost $31 \%$ of patients exhibited nodal involvement while distant metastasis was observed in $7 \%$ of cases. Out of 47 cases in grade III, only one showed a low Ki-67 index while only $12 \%$ patients in grade II exhibited a similar trend. The odds of having a high Ki-67 index were significantly high in IDC as compared to the non-IDC cases. Besides IDC, all three cases of ILC were negative for Ki-67 having luminal A subtype while all three metaplastic cases exhibited a high Ki-67 index. Almost all patients presenting with stage 3 (95\%) and stage 4 (100\%) showed a higher Ki-67 index. Interestingly, $84 \%$ of the patients in initial stages (I \& II) were also found to have Ki-67>14\%, showing a greater frequency of proliferation marker in the cohort (Table 1). A higher mitotic index was observed in patients having the triple negative (40\%) and HER2 (34\%) subtype.

Association of Ki-67 with Age and Menopausal Status

Almost 89 and $88 \%$ of patients were found to have a high Ki-67 index in $\leq 47$ and $>47$ years of age groups, respectively. Therefore, a significant difference was not observed between the two groups. A significant number a

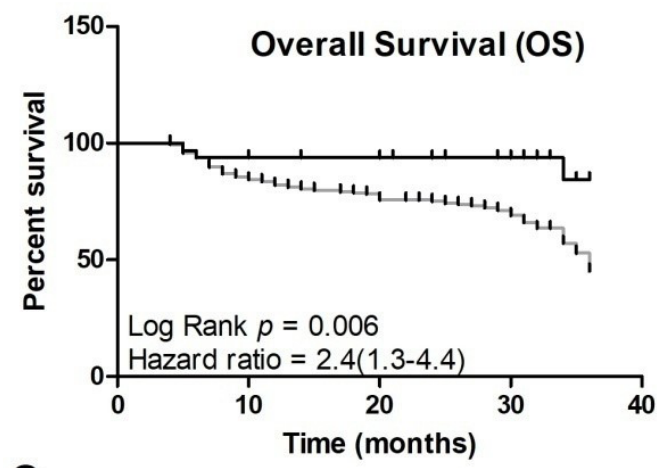

C

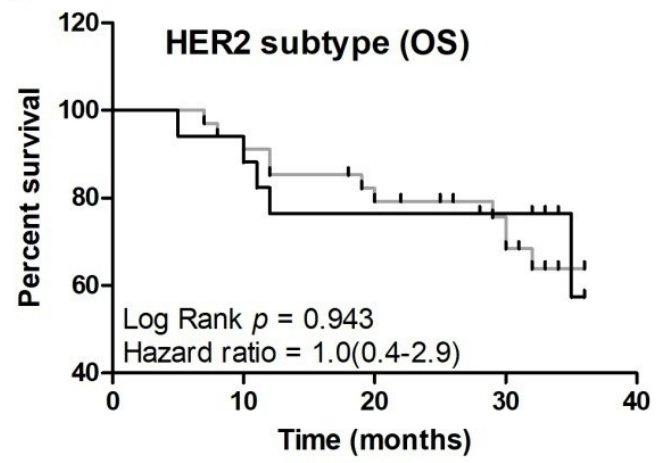

b
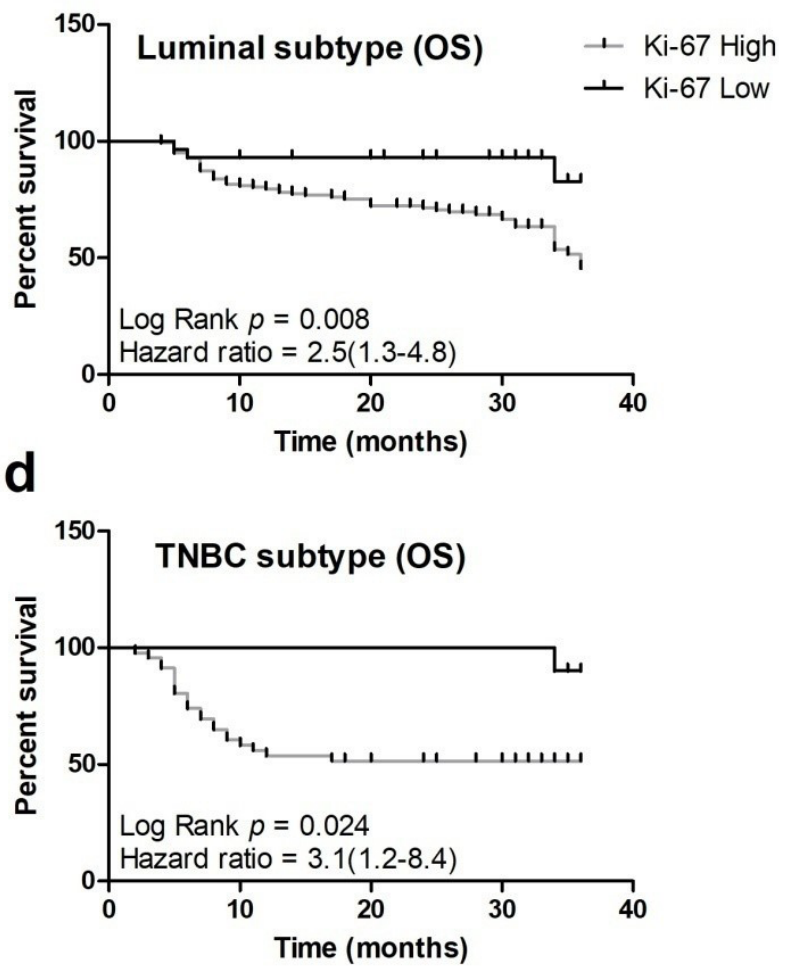

Figure 1. Frequency of high and low Ki-67 expression in (a) ER positive and negative (b) PR positive and negative (c) HER2 positive and negative and (d) patients categorized in different molecular subtypes (** represents $P<0.01$, *** represents $P<0.0001$ ). 


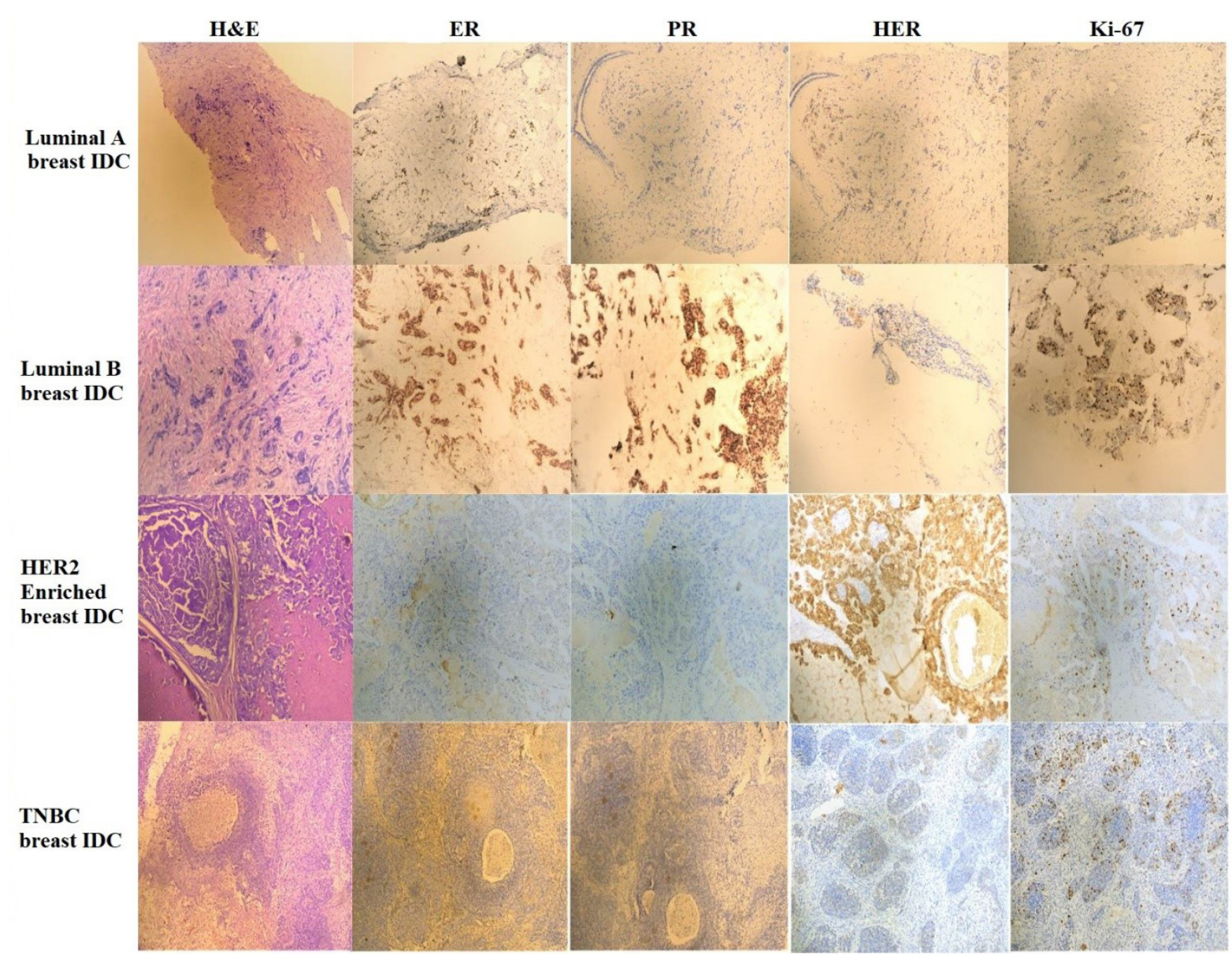

Figure 2. Photomicrographs of H\&E Staining, ER, PR, HER2 and Ki-67 Immunostaining in Patients Belonging to Luminal A, Luminal B, HER2 Enriched and TNBC Subtypes, Respectively.

of patients $(70 \%)$ in the triple negative group exhibited early onset. On the contrary, almost $60 \%$ of patients in the luminal A and HER2 enriched subtype presented with late disease onset. In the premenopausal group, 90\% of patients were found to have Ki-67 > 14\% while $85 \%$ of postmenopausal patients also showed similar results. The majority of premenopausal patients were found to have the luminal B (61\%) and triple negative (82\%) subtype intrinsically (Tables 1 and 2).

\section{Relationship of Ki-67 with Survival}

The follow-up period for the study was about 3 years with an overall survival rate of $64 \%$. Interestingly, $97 \%$ of the patients having a high Ki-67 index did not survive during the follow-up period (Table 1). A high Ki-67 index proved to be a significant marker of poor prognosis in breast cancer affected patients $(P=0.006)$. In the high Ki-67 group, 97 patients died while 148 patients were censored as they were alive when the follow-up period of the study terminated. The majority of patients having luminal B (40\%), HER2 (50\%) and triple negative (41\%) demonstrated poor mortality rate due to aggressive features (Figure 3, Table 2).

\section{Discussion}

Breast cancer is a heterogeneous disease presenting with diverse clinical and histological features. Based on ER, PR, HER-2 and Ki-67 immunostains, the tumors are classified into four molecular subtypes with distinctive tumor characteristics, prognosis and treatment response. Several studies have shown Ki-67 as an independent predictor for pathological response in patients amongst all molecular subtypes. ${ }^{13} \mathrm{Ki}-67$ is considered as an important factor in relation to neoadjuvant chemotherapy in all molecular subtypes. ${ }^{14}$ The luminal A type benefits from hormonal therapy and is less responsive to chemotherapy. ${ }^{15}$ However, chemotherapy can be added for luminal A patients based on large tumor size and other risk factors. The higher the $\mathrm{Ki}-67$ is, the more responsive the cancer is to chemotherapy. Ki-67 is also one of the genes included in the Oncotype DX prognostic score which includes a total of 21 genes. The Oncotype Dx score helps in predicting the benefit of chemotherapy and risk of recurrence. As observed in our study, Ki-67 can have potential use in determining relative prognosis, resistance to endocrine therapy or chemotherapy and estimation of residual risk. ${ }^{16}$

The majority of patients (68\%) in our study were between 40 and 60 years of age. Mean age was 47.9 years which is concordant with a local study done in Pakistan. ${ }^{17}$ In this study, the population was relatively younger than Western countries. In contrast to developing countries, in the United States and other developed countries, the 
Table 1. Association of Ki-67 with Demographic and Clinical Features

\begin{tabular}{|c|c|c|c|c|c|c|c|}
\hline Parameters Observed & & $\begin{array}{c}\text { Total } \\
(n=278)\end{array}$ & Percentage $(\%)$ & Ki-67 High & Ki-67 Low & $\begin{array}{c}\text { Odds ratio } \\
(95 \% \text { Confidence Interval) }\end{array}$ & $P$ Value \\
\hline \multirow[t]{2}{*}{ ER } & ER Positive & 169 & 61 & 141 & 28 & $0.2(0.1-0.6)$ & $0.002^{*}$ \\
\hline & ER Negative & 109 & 39 & 104 & 5 & & \\
\hline \multirow[t]{2}{*}{$P R$} & PR Positive & 129 & 46 & 106 & 23 & $0.3(0.2-0.7)$ & $0.005^{*}$ \\
\hline & PR Negative & 149 & 54 & 139 & 10 & & \\
\hline \multirow[t]{2}{*}{ HER2 } & HER-2 Positive & 138 & 50 & 133 & 5 & $6.6(2.5-17.8)$ & $<0.0001^{*}$ \\
\hline & HER-2 Negative & 140 & 50 & 112 & 28 & & \\
\hline \multirow[t]{4}{*}{ Molecular subtype } & Luminal A & 29 & 11 & 0 & 29 & - & $<0.0001$ \\
\hline & Luminal B & 142 & 51 & 142 & 0 & & \\
\hline & HER2 & 51 & 18 & 50 & 1 & & \\
\hline & TNBC & 56 & 20 & 53 & 3 & & \\
\hline \multirow[t]{2}{*}{ Age } & $\leq 47$ & 141 & 51 & 125 & 16 & $1.1(0.5-2.3)$ & $0.85^{*}$ \\
\hline & $>47$ & 137 & 49 & 120 & 17 & & \\
\hline \multirow[t]{2}{*}{ Menopausal status } & Pre-Menopausal & 172 & 62 & 155 & 17 & $1.6(0.8-3.4)$ & $0.25 *$ \\
\hline & Post-Menopausal & 106 & 38 & 90 & 16 & & \\
\hline \multirow[t]{3}{*}{ Histopathological type } & IDC & 272 & 98 & 242 & 30 & - & $<0.0001$ \\
\hline & ILC & 3 & 1 & 0 & 3 & & \\
\hline & Metaplastic & 3 & 1 & 3 & 0 & & \\
\hline \multirow[t]{2}{*}{ IDC vs non-IDC } & IDC & 272 & 98 & 242 & 30 & $8(1.6-41.8)$ & $0.02 *$ \\
\hline & Non-IDC & 6 & 2 & 3 & 3 & & \\
\hline \multirow[t]{3}{*}{ Grade } & Grade 1 & 42 & 15 & 34 & 8 & - & 0.003 \\
\hline & Grade 2 & 188 & 68 & 164 & 24 & & \\
\hline & Grade 3 & 48 & 17 & 47 & 1 & & \\
\hline \multirow[t]{4}{*}{ Stage } & Stage 1 & 41 & 15 & 36 & 5 & - & 0.01 \\
\hline & Stage 2 & 127 & 46 & 104 & 23 & & \\
\hline & Stage 3 & 90 & 32 & 85 & 5 & & \\
\hline & Stage 4 & 20 & 7 & 20 & 0 & & \\
\hline \multirow[t]{3}{*}{ Nodal/distant Metastasis } & No Metastasis & 171 & 62 & 143 & 28 & - & 0.01 \\
\hline & Nodal Invasion & 87 & 31 & 82 & 5 & & \\
\hline & Distant Metastasis & 20 & 7 & 20 & 0 & & \\
\hline \multirow[t]{2}{*}{ Survival Status } & Alive & 178 & 64 & 148 & 30 & $0.2(0.04-0.5)$ & $0.0004^{*}$ \\
\hline & Death & 100 & 36 & 97 & 3 & & \\
\hline
\end{tabular}

*Fisher Exact test.

average age at presentation is 60 years. ${ }^{18}$

The majority of patients in this study belonged to grade II which is in line with local and international studies. ${ }^{17}$ The current study has shown that increasing grade is associated with higher proliferation depicted by a high Ki-67 value, as shown by $98 \%$ cases of Grade III having high Ki-67 (>14\%) followed by $87 \%$ and $81 \%$ of grade II and grade I of breast cancers. Previously, it has been observed that $70 \%, 64 \%$ and $42 \%$ of grade I, grade II and grade III tumors respectively, have high Ki-67 (>14\%). ${ }^{19}$ This is supported by the results of a German cohort with a significant correlation between high Ki-67 and high-grade tumors. ${ }^{20}$

Furthermore, a high Ki-67 index was established as an independent prognostic as well as predictive marker in the breast cancer cohort, exerting effect on overall survival (OS). Similarly, a Ki-67 index > 15 was found to be a marker of poor prognosis in the German cohort. ${ }^{20}$ Besides the luminal B subtype, a high Ki-67 index was also associated with poor survival in triple negative patients in the present cohort. On the contrary, no correlation was observed between the effect of a high Ki-67 index with patients belonging to the HER-2 enriched subtype and overall survival. Coherently, a high Ki-67 index was found to be associated with ER-ve, PR-ve and HER-ve patients and not HER-2 positive patients in an Egyptian study. ${ }^{21}$

Estrogen positive subtypes (luminal type A and luminal type B) collectively constitute the majority of the breast cancer burden observed in our study (ER positive 61\% cases). Estrogen is not only involved in embryogenesis of the mammary gland, but also significantly modulates the effect of anti-estrogen therapy through intricate molecular pathways. The luminal group is markedly heterogenous with complex cellular signalling pathways involving ER 
Table 2. Distribution of Study Samples According to Molecular Subtypes for Frequency of Ki-67 immunoexpression

\begin{tabular}{|c|c|c|c|c|c|c|}
\hline & & Luminal A & Luminal B & HER2 & TNBC & $P$ Value \\
\hline \multirow[t]{2}{*}{ Ki-67 } & Ki-67 Positive & 0 & 142 & 50 & 53 & $<0.0001$ \\
\hline & Ki-67 Negative & 29 & 0 & 1 & 3 & \\
\hline \multirow[t]{2}{*}{ ER } & ER Positive & 28 & 141 & 0 & 0 & $<0.0001$ \\
\hline & ER Negative & 1 & 1 & 51 & 56 & \\
\hline \multirow[t]{2}{*}{ PR } & PR Positive & 23 & 106 & 0 & 0 & $<0.0001$ \\
\hline & PR Negative & 6 & 36 & 51 & 56 & \\
\hline \multirow[t]{2}{*}{ HER2 } & HER-2 Positive & 4 & 83 & 51 & 0 & $<0.0001$ \\
\hline & HER-2 Negative & 25 & 59 & 0 & 56 & \\
\hline \multirow[t]{2}{*}{ Age } & $\leq 47$ & 12 & 70 & 20 & 39 & 0.008 \\
\hline & $>47$ & 17 & 72 & 31 & 17 & \\
\hline \multirow[t]{2}{*}{ Menopausal status } & Pre-Menopausal & 13 & 87 & 26 & 46 & 0.001 \\
\hline & Post-Menopausal & 16 & 55 & 25 & 10 & \\
\hline \multirow[t]{3}{*}{ Histopathological type } & IDC & 26 & 141 & 50 & 55 & $<0.0001$ \\
\hline & ILC & 3 & 0 & 0 & 0 & \\
\hline & Metaplastic & 0 & 1 & 1 & 1 & \\
\hline \multirow[t]{2}{*}{ IDC vs Non-IDC } & IDC & 26 & 141 & 50 & 55 & 0.01 \\
\hline & Non-IDC & 3 & 1 & 1 & 1 & \\
\hline \multirow[t]{3}{*}{ Grade } & Grade 1 & 8 & 25 & 1 & 8 & 0.001 \\
\hline & Grade 2 & 20 & 99 & 37 & 32 & \\
\hline & Grade 3 & 1 & 18 & 13 & 16 & \\
\hline \multirow[t]{4}{*}{ Stage } & Stage 1 & 5 & 19 & 10 & 7 & 0.28 \\
\hline & Stage 2 & 19 & 62 & 23 & 23 & \\
\hline & Stage 3 & 5 & 50 & 13 & 22 & \\
\hline & Stage 4 & 0 & 11 & 5 & 4 & \\
\hline \multirow[t]{3}{*}{ Nodal/distant Metastasis } & No Metastasis & 24 & 83 & 34 & 30 & 0.12 \\
\hline & Nodal Invasion & 5 & 48 & 12 & 22 & \\
\hline & Distant Metastasis & 0 & 11 & 5 & 4 & \\
\hline \multirow[t]{2}{*}{ Survival Status } & Alive & 26 & 85 & 34 & 33 & 0.01 \\
\hline & death & 3 & 57 & 17 & 23 & \\
\hline
\end{tabular}

mediating subunit 1 (MED 1) and HER-2 co-amplification influencing treatment effects. ${ }^{22}$ In the present study, luminal B comprised $51 \%$ of cases which is comparable to a study done in the Republic of Moldova with $40 \%$ luminal B cases. ${ }^{2,4}$ Our study showed $10 \%$ of cases falling into the luminal A subtype which is in accordance with Petric et al with $12.5 \%$ cases having the luminal A subtype. ${ }^{23}$ Gogoi et al showed $19 \%$ cases of luminal A which is close to this study. ${ }^{24}$ Most of the luminal A subtype patients are of low grade and this trend has been reported in a study done on cases obtained from Chicago care. ${ }^{6}$ Both luminal subtypes are responsive to hormonal therapy. Studies have shown that discriminating luminal A from luminal B is important from the prognostic point of view. Luminal A commonly recurs in bone and rarely metastasizes to visceral organs, whereas luminal B has higher chances of visceral with or without bone metastases. The more likely reason for the low incidence of luminal A in our study is lack of proper screening and public awareness. Similarly, the results of Gogoi et al showed HER2 enriched cases accounting for $17.88 \%$, which is in line with this study where HER2 enriched comprised $18 \%$ of cases. ${ }^{24}$ The present study showed most of HER2 enriched (31\%) breast cancers to be more prevalent in the age group above 47 years. This is contrary to a study done by Anderson et al where most of the HER 2 enriched subtype pertained to those under 40 years of age. ${ }^{6}$ A possible explanation for these findings is higher alertness around menopause and more density on mammogram in the young age which could be areas on for late presentation and progressive stage.

Due to the multifactorial nature of the disease, there are certain limitations regarding confounders including comorbidities, lifestyle, occupational hazards and obesity for which the information was unavailable. Therefore, these factors could not be included in the multivariable analysis. Moreover, the possibility of sparse-data bias can also be considered as a limitation of the study. ${ }^{25}$

IHC is an easy and cost-effective technique compared to more sophisticated techniques like DNA sequencing which is expensive and not applicable in routine clinical 


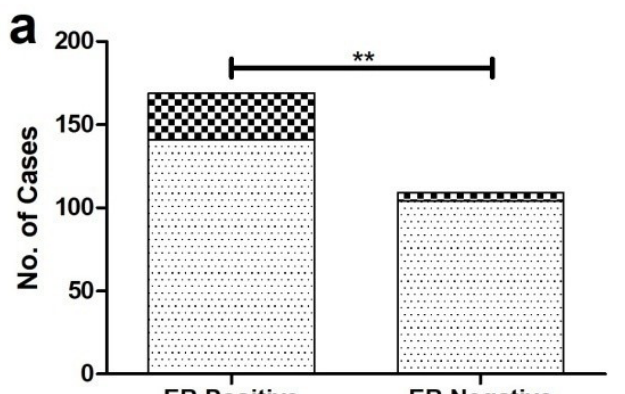

ER Positive b

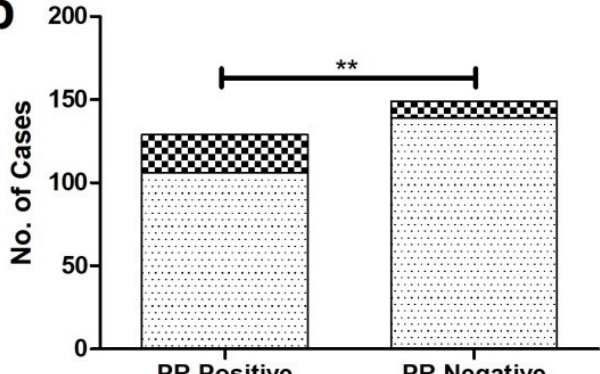

PR Positive
Ki-67 High

\$. Ki-67 Low
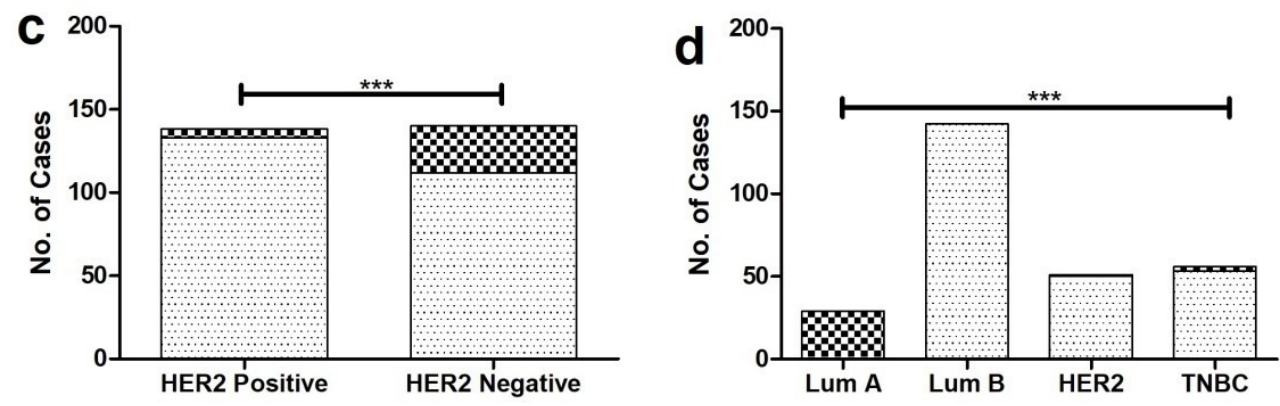

Figure 3. Kaplan-Meier Plots Showing Association of Ki-67 Index with Patient's (a). Overall survival (OS) (b). OS in Luminal subtypes (c). OS in HER2 enriched subtype (d). OS in Triple negative subtype in the cohort $(P<0.05$ is considered significant).

pathology. A cut-off threshold of $14 \%$ for the Ki-67 index proved to be a valuable predictive and prognostic marker for influencing therapeutic and clinical decisions. Hence, there is an urgent need for establishing an effective screening program using IHC4 (ER, PR, HER-2 and Ki67 ) at the national level for early detection of cases which will help in treatment and subsequently, improve patient prognosis.

\section{Availability of data}

Data regarding individual staining results can be furnished upon request.

\section{Authors' Contribution}

MM and SS; Conceptualization, investigation, methodology, writing - original draft, writing - review \& editing, data curation. AKS; Formal analysis, validation, supervision, writing - review \& editing. NK; Conceptualization, resources. AK; Project administration, conceptualization, funding acquisition. AA; Formal analysis, investigation, methodology.TK and AKT, Formal analysis, validation, supervision, conceptualization, data curation, writing - review \& editing. MFAM, Funding acquisition, investigation, methodology. SKR; Formal analysis, software, writing - original draft, writing review \& editing, funding acquisition, investigation, methodology. Competing interests

The authors of the study have no conflict of interest.

\section{Ethics Statement}

The study proceeded with prior approvals from biosafety and ethical committees of both Shaheed Zulfiqar Ali Bhutto Medical University and Pakistan Institute of Medical Sciences (PIMS) hospital. Informed consents from the participants were collected after a thorough briefing of the proposed research.

\section{Funding}

The present work was partially funded by grant no. SRGP-2603 from Higher Education commission, Pakistan.

\section{Acknowledgements}

We are grateful to all participants involved in this study.

\section{References}

1. Ferlay J, Soerjomataram I, Dikshit R, Eser S, Mathers C, Rebelo $\mathrm{M}$, et al. Cancer incidence and mortality worldwide: sources, methods and major patterns in GLOBOCAN 2012. Int J Cancer. 2015;136(5):E359-86. doi: 10.1002/ijc.29210.

2. Fulga V, Rudico L, Balica AR, Cimpean AM, Saptefrati L, Raica M. Invasive ductal carcinoma of no special type and its corresponding lymph node metastasis: do they have the same immunophenotypic profile? Pol J Pathol. 2015;66(1):30-7. doi: 10.5114/pjp.2015.51150.

3. Abubakar M, Figueroa J, Ali HR, Blows F, Lissowska J, Caldas C, et al. Combined quantitative measures of ER, PR, HER2, and Ki67 provide more prognostic information than categorical combinations in luminal breast cancer. Mod Pathol. 2019;32(9):1244-56. doi: 10.1038/s41379-019-02704.

4. Paramita S, Raharjo EN, Niasari M, Azizah F, Hanifah NA. Luminal $B$ is the most common intrinsic molecular subtypes of invasive ductal breast carcinoma patients in East Kalimantan, Indonesia. Asian Pac J Cancer Prev. 2019;20(8):2247-52. doi: 10.31557/apjcp.2019.20.8.2247.

5. Pandit P, Patil R, Palwe V, Gandhe S, Patil R, Nagarkar R. Prevalence of molecular subtypes of breast cancer: a single institutional experience of 2062 patients. Eur J Breast Health. 2020;16(1):39-43. doi: 10.5152/ejbh.2019.4997.

6. Anderson WF, Rosenberg PS, Prat A, Perou CM, Sherman ME. How many etiological subtypes of breast cancer: two, three, four, or more? J Natl Cancer Inst. 2014;106(8):dju165. doi: 10.1093/jnci/dju165.

7. Stathopoulos GP, Malamos NA, Markopoulos C, Polychronis A, Armakolas A, Rigatos S, et al. The role of $\mathrm{Ki}-67$ in the proliferation and prognosis of breast cancer molecular classification subtypes. Anticancer Drugs. 2014;25(8):950-7. doi: 10.1097/cad.0000000000000123.

8. Kadivar M, Mafi N, Joulaee A, Shamshiri A, Hosseini N. Breast cancer molecular subtypes and associations with 
clinicopathological characteristics in Iranian women, 20022011. Asian Pac J Cancer Prev. 2012;13(5):1881-6. doi: 10.7314/apjcp.2012.13.5.1881.

9. Dowsett M, Nielsen TO, A'Hern R, Bartlett J, Coombes RC, Cuzick J, et al. Assessment of Ki67 in breast cancer: recommendations from the International Ki67 in breast cancer working group. J Natl Cancer Inst. 2011;103(22):1656-64. doi: 10.1093/jnci/djr393.

10. Ahmed ST, Ahmed AM, Musa DH, Sulayvani FK, Al-Khyatt M, Pity IS. Proliferative index (Ki67) for prediction in breast duct carcinomas. Asian Pac J Cancer Prev. 2018;19(4):955-9. doi: 10.22034/apjcp.2018.19.4.955.

11. Goldhirsch A, Wood WC, Coates AS, Gelber RD, Thürlimann B, Senn HJ. Strategies for subtypes--dealing with the diversity of breast cancer: highlights of the St. Gallen International Expert Consensus on the Primary Therapy of Early Breast Cancer 2011. Ann Oncol. 2011;22(8):1736-47. doi: 10.1093/ annonc/mdr304.

12. Cho SY, Park SY, Bae YK, Kim JY, Kim EK, Kim WG, et al. Standardized pathology report for breast cancer. J Pathol TransI Med. 2021;55(1):1-15. doi: 10.4132/jptm.2020.11.20.

13. Abubakar M, Orr N, Daley F, Coulson P, Ali HR, Blows F, et al. Prognostic value of automated Ki67 scoring in breast cancer: a centralised evaluation of 8088 patients from 10 study groups. Breast Cancer Res. 2016;18(1):104. doi: 10.1186/s13058016-0765-6.

14. Zhu X, Chen L, Huang B, Wang Y, Ji L, Wu J, et al. The prognostic and predictive potential of Ki-67 in triple-negative breast cancer. Sci Rep. 2020;10(1):225. doi: 10.1038/s41598019-57094-3.

15. Goldhirsch A, Winer EP, Coates AS, Gelber RD, PiccartGebhart M, Thürlimann B, et al. Personalizing the treatment of women with early breast cancer: highlights of the St. Gallen International Expert Consensus on the Primary Therapy of Early Breast Cancer 2013. Ann Oncol. 2013;24(9):2206-23. doi: 10.1093/annonc/mdt303.

16. Thakur SS, Li H, Chan AMY, Tudor R, Bigras G, Morris D, et al. The use of automated Ki67 analysis to predict Oncotype DX risk-of-recurrence categories in early-stage breast cancer. PLoS One. 2018;13(1):e0188983. doi: 10.1371/journal. pone.0188983.

17. Al-Mahmood S, Sapiezynski J, Garbuzenko OB, Minko T. Metastatic and triple-negative breast cancer: challenges and treatment options. Drug Deliv Transl Res. 2018;8(5):1483507. doi: 10.1007/s13346-018-0551-3.

18. Nekhlyudov L, Mollica MA, Jacobsen PB, Mayer DK, Shulman LN, Geiger AM. Developing a quality of cancer survivorship care framework: implications for clinical care, research, and policy. J Natl Cancer Inst. 2019;111(11):1120-30. doi: 10.1093/jnci/djz089.

19. Sun J, Chen C, Wei W, Zheng H, Yuan J, Tu YI, et al. Associations and indications of Ki67 expression with clinicopathological parameters and molecular subtypes in invasive breast cancer: a population-based study. Oncol Lett. 2015;10(3):1741-8. doi: 10.3892/ol.2015.3461.

20. Zhao X, Yang X, Fu L, Yu K. Associations of estrogen receptor, progesterone receptor, human epidemic growth factor receptor-2 and Ki-67 with ultrasound signs and prognosis of breast cancer patients. Cancer Manag Res. 2021;13:4579-86. doi: $10.2147 / \mathrm{cmar} . \mathrm{s} 276422$.

21. Soliman NA, Yussif SM. Ki-67 as a prognostic marker according to breast cancer molecular subtype. Cancer Biol Med. 2016;13(4):496-504. doi: 10.20892/j.issn.20953941.2016.0066.

22. Leonard M, Zhang X. Estrogen receptor coactivator Mediator Subunit 1 (MED1) as a tissue-specific therapeutic target in breast cancer. J Zhejiang Univ Sci B. 2019;20(5):381-90. doi: 10.1631/jzus.B1900163.

23. Petric M, Martinez $S$, Acevedo F, Oddo D, Artigas R, Camus $M$, et al. Correlation between Ki67 and histological grade in breast cancer patients treated with preoperative chemotherapy. Asian Pac J Cancer Prev. 2014;15(23):10277-80. doi: 10.7314/ apjcp.2014.15.23.10277.

24. Gogoi G, Borgohain M, Saikia P, Fazal SA. Profile of molecular subtypes of breast cancer with special reference to triple negative: a study from Northeast India. Clin Cancer Investig J. 2016;5(5):374-83. doi: 10.4103/2278-0513.197871.

25. Greenland S, Mansournia MA, Altman DG. Sparse data bias: a problem hiding in plain sight. BMJ. 2016;352:i1981. doi: 10.1136/bmj.i1981. 